\title{
Oncogenic Osteomalacia: A New Observation
}

\section{N. Boussetta, S. Hamrouni, Rim Dhahri, N. Gueddiche, F. Ajili, B. Louzir}

Internal Medicine Deaprtment, Military Hospital of Tunis, Tunisia. toile_du_nord@yahoo.fr

*Corresponding Author: Najah Boussetta, Internal Medicine Deaprtment, Military Hospital of Tunis, Tunisia.

\section{Abstract}

Oncogenic osteomalacia is a rare paraneoplastic syndrome resulting from an increased secretion of a fibroblast growth factor 23 (FGF23), generally by a benign mesenchymal tumor. We report a case of a 60 years old female patient who had chronic bone pain. A Diagnosis of osteomalacia was made based on the following: difficulty walking, waddling gait, bone pain, a normal rate of calcemia PTH and of $25 \mathrm{OH}$ vitamin D3 associated with an increased alkaline phosphatase rate, hypophosphoremia and a decrease in the rate of tubular reabsorption of phosphates. The neoplastic origin was suspected in the presence of a biological inflammatory syndrome and was confirmed by a high level of FGF23. The tumor could not be located and the patient received calcitriol and phosphorus

\section{INTRODUCTION}

Tumor-induced osteomalacia, also known as oncogenic osteomalacia (00), is a rare paraneoplastic syndrome caused by the overproduction of fibroblast growth factor 23 (FGF23) by a benign and small-sized mesenchymal tumor. This syndrome results from a decrease in the 1-hydroxylation capacity of the 25 hydroxy-vitamin Dand a hypophosphatemiasecondary to a urinary phosphate leak in which the FGF23 plays an essential role. To date, around 100 cases have been published. We report a new observation of a patient with 00.

\section{CASE REPORT}

A 60-year-old woman was hospitalized after the discovery of hypophosphatemia in the exploration of bone pain in her hips and left shoulder which had been present for two years. The patient did not claim a history of alcohol or tobacco use, and took no medications other than painkillers. There was no reported family history of osteoarticular disease. The patient had a waddling gait and painful mobility of both hips and in the left shoulder. Calcium, PTH and $25 \mathrm{OH}$ vitamin D3 levels were normal with increased alkaline phosphatase levels and hypophosphoremia to $0.17 \mathrm{mmol} / \mathrm{L}$ with a marked decrease in the tubular reabsorption rate of phosphates at 71\% (N> 85\%). Abiological inflammatory syndrome was present as evidenced byX-rays which revealed hypertransparency with blurred bone, Looser-Milkman streaks at the ribs and neck of the left femur, and biconcave vertebrae.

The patient had osteoporosis with a T-score to -4.4.Scintigraphy revealed numerous foci of hyperfixation in the spine, the costal cartilidge, the left acetabulum and the right femur. The diagnosis of osteomalacia was confirmed based on the normal rate of vitamin D and PTH and the presence of a biological inflammatory syndrome, the origin of which was the most likely paraneoplastic. The concentration of FGF-23 was abnormally high at $284 \mathrm{Ru} / \mathrm{ml}$ (44$140 \mathrm{Ru} / \mathrm{ml}$ ). In addition, The concentration of chromogranin A and neuro-specific enolase was high. The thoracoabdominopelvic CT scan revealed an mesenteric adenomegaly. Ostreoscan scintigraphy did not show a tumor. Positron emission tomography (PET) imaging with $18 \mathrm{~F}$-based radiotracers showed two hypermetabolic adenomegalies with a pathological hypermetabolic focus of the last ileal loop without structural abnormality and a diffuse hypermetabolic focus of the right foot muscles. The ileal biopsy was normal. Mesenteric adenomegalies were difficult to access. A clinical improvement was obtained by daily intake of 0.5 microgram daily calcitriol (2cps Rocaltrol $($ ) and $1500 \mathrm{mg}$ oral phosphorus (200 drops of Phosphoneuros ${ }^{\circledR}$ ). The search for the tumor in question is still in progress. 


\section{DISCUSSION}

00 is a rare acquired pathology. In the literature, just over 160 cases have been reported [1]. Few studies have discussed the incidence and prevalence of 00 . It usually occurs in adults with an average age of 45 years. Few cases have been reported in children [2]. There is no difference in incidence between the two age groups. Clinical manifestations such as fatigue, pain and bone fractures, as well as prolonged hypophosphatemia, are not exclusive to $00[2]$. The clinical signs and symptoms of oncogenic osteomalacia as a neoplastic syndrome often lead to a misdiagnosis of joint, muscle or neurological pathologies. Most patients report fatigue, bone or muscle pain [3]. Our patient reported pain in both hips and the left shoulder which had been evolving for two years.

Biochemical markers of 00 include hypophosphatemia, hyperphosphaturia, decreased tubular phosphate reabsorption, elevated rate of alkaline phosphatase associated with normal levels of calcium, $25(\mathrm{OH})$ vitamin D, and PTH rate. The responsibility of FGF 23 in the occurrence of these biological abnormalities has been demonstrated in 00s. High levels of FGF23 lead to a decrease in phosphate reabsorption in the proximal convoluted tubule by inhibition of the sodium-phosphate co-transporter, and independently inhibit renal expression of 25-hydroxyvitamin D-1alpha-hydroxylase. High serum FGF 23 levels are thus found in the greatest number of 00s. Tumors secreting FGF23 are usually benign [4]. The most frequently identified neoplasms are vascular neoplasia but also chondrosarcomas, osteosarcomas, histiocytomas, fibroids, neuroblastomas and giant cell tumors [1]. They are usually small and explain the long diagnostic delay. The most effective treatment of 00 is the excision of the tumor, which allows rapid and lasting normalization of biological and clinical signs. Nevertheless, some tumors remain non-localized as in the case of our patient [5]. A medical treatment based on oral phosphate supplementation associated with calcium and vitamin D allows a clinical improvement.

\section{ConCLUSION}

00 is a rare pathology. It combines severe hypophosphatemia, hyperphosphaturia and a very low blood concentration of $1,25-(\mathrm{OH})$ vitamin $\mathrm{D}$. The increase in FGF 23 rate confirms the diagnosis. The best treatment is excision of the tumor. Nevertheless, in the case of non-localized tumors, medical treatment with phosphate and calcitriol allows a biological and clinical improvement.

\section{REFERENCES}

[1] C. van der Rest E. Cavalier L. Colson et al. Hypophosphatémieetostéomalacie oncogénique. Rev Med Suisse 2011; 7: 1630-3

[2] Subramanian R, Khardori R. Severe hypophosphatemia: pathophysiologic implications, clinical presentations and treatment. Medicine 2000;79:1-8

[3] R.M. Radaideh, D. Jaradat, M.M. Abu-Kalaf, and M.K. Nusier. Resolution of severe oncogenic hypophosphatemic osteomalacia after resection of a deeply located soft-tissue tumour. Curr Oncol $6(5)$

[4] Seufert J, Ebert K, Muller J, et al. Octreotide therapy for tumor-induced osteomalacia. N Engl J Med 2001;345:1883-8

[5] Geller JL, Khosravi A, Kelly MH, et al. Cinacalcet in the management of tumor-induced osteomalacia. J Bone Miner Res 2007;22:931-7

Citation: N. Boussetta, S. Hamrouni, Rim Dhahri, N. Gueddiche, F. Ajili, B. Louzir. Oncogenic Osteomalacia: A New Observation. Open Access Journal of Internal Medicine. 2018; 1(1): 41-42.

Copyright: (C) 2018 N. Boussetta, S. Hamrouni, Rim Dhahri, N. Gueddiche, F. Ajili, B. Louzir. This is an open access article distributed under the Creative Commons Attribution License, which permits unrestricted use, distribution, and reproduction in any medium, provided the original work is properly cited. 